\title{
Line Drawing Interpretation Using Belief Propagation
}

\author{
Yansheng Ming \\ School of Engineering \\ Australian National University \\ Canberra, Australia 0200 \\ Email: yansheng.ming@anu.edu.au
}

\author{
Hongdong Li \\ School of Engineering \\ Australian National University \\ Canberra, Australia 0200 \\ Email: hongdong.li@anu.edu.au
}

\author{
Jun Sun \\ School of Engineering \\ Australian National University \\ Canberra, Australia 0200 \\ Email: sunjun1104@gmail.com
}

\begin{abstract}
The interpretation of line drawings of trihedral planer objects is a classic problem. In this paper, it is formulated as a Bayesian inference problem. Given a line drawing image, a Markov random field can be built whose nodes represent the labels of edges. Its clique potential functions are designed to encode the valid junctions in the Huffman-Clowes catalogue. The belief propagation algorithm is used to find the most probable labeling of the edges. We find this algorithm closely related to the arc consistency methods. However our probabilistic formulation can accommodate uncertainty in junction detection and make use of various image cues. These advantages are demonstrated in the experiments.
\end{abstract}

Keywords-line drawing image labeling; Markov random field; belief propagation

\section{INTRODUCTION}

Line drawing interpretation is one of the problems where 3D information is inferred from a $2 \mathrm{D}$ image. Unlike the problem of single image depth estimation [1], 3D information in the line drawings is highly structured and sparse. Huffman and Clowes [2][3] studied the line drawings of trihedral objects (degree-3 polyhedrons). Interestingly, they found that among all the possible labeling of a junction, which is the image of a vertex of the trihedral object, only a few of them are valid. In other words, the types of the junctions and their connections largely determined 3D information in the image. The immediate problem is how to find a valid labeling of edges that satisfies the constraints of all the junctions. Traditionally, this problem has been formulated as a constraint satisfying problem. The arc consistency algorithms can efficiently find a solution if the junctions are correctly detected. However, perfect detection may not be possible for natural images. Some important edges may be missed by the edge detection algorithm such that the image becomes impossible to label. On the other hand, various local cues in natural images may be valuable for labeling the image. For example, an edge has high probability to be an occluding contour if the texture differs greatly on both sides. These situations call for a probabilistic treatment of the classic problem because they are outside the scope of deterministic formulation.

In this paper, the problem of the line drawing labeling is modeled as a Markov random field (MRF) inference problem. In this model, each node is associated with an edge in the image. There are four labels of each node, representing convex edge, concave edge, and occluding edges with two possible directions. There is one clique for every junction. Each clique potential is customized to encode one of the four junction types: 'L','W', 'Y','T'. There are also parameters to control the uncertainty about the junction. If a junction is noisy, its potential function can be adapted to assign a moderate probability value to invalid configurations. Besides, there is one likelihood term for every edge, encoding information of other image cues. Ideally, these potential functions should be obtained from classifiers trained on a set of natural images containing polyhedra. In this paper, these likelihood functions are set by hand. However, their effectiveness is demonstrated in the experiment.

The correctness of the belief propagation algorithm (BP) on a general Markov random field is still an open problem, although considerable progress has been made [4]. In our experiment, BP converges quickly and generally leads to right solutions. We would like to indicate the similarity of BP and methods based on arc consistency. When all the potential functions are binary, the message passing in BP is quite similar, if not identical, to the propagation of constraints in the arc consistency methods. This could serve as an explanation to BPs success. However, BP can propagate probabilistic information in addition to hard constraints.

\section{RELATED WORK}

In any image of trihedral objects taken at a general view point, the junctions can be classified as the following four types: 'L', 'W', 'Y', and 'T'. Huffman and Clowes enumerated every possible labeling for each of these junction types, and they are shown in Figure 1. Note that after an arbitrary rotation, each junction in Figure 1 remains valid. Still, the number of valid labeling are very small compared with all the possible ways of labeling. For example, only 3 out of $64 \mathrm{~W}$ junctions are valid for trihedral objects.

Based on these local constraints, Waltz [5][6] and Mackworth [6][7][8] developed a constraint propagation algorithm. The main idea is to consider a label infeasible (and be removed from the junction) if it is not consistent with any feasible labels of its adjacent nodes. When one label is removed at a junction, the algorithm will check feasibility of labels of the neighboring 
nodes. This algorithm was later analyzed in the frame work of arc consistency. The algorithms based on the theory of arc consistency can efficiently solve the problem in practice.

Regier [9] formulated line drawing labeling as a coupled MRF. His model tried to infer the type of junctions along with the labels of edges. However, this made his model so complicated that he had to adopt a collapsed label set in which the directions of occluding edge are not differentiated. In our model, the junction types are predetermined; however, the potentials at each junction can adapt to uncertainty in junction detection phase.

A lot of works has been done to extend the scope to nontrihedral objects. For example, the catalogue of valid junctions of 4-hedron has been built. Similar catalogue for smooth objects with curved contours also has been proposed [10][11]. In a broad sense, understanding the line drawings is more than assigning these labels to contours. For example, some researchers tried to find parallel lines in the line drawings [12]. Probably, the ultimate goal is to build $2.5 \mathrm{D}$ or $3 \mathrm{D}$ representation [13] from the line drawing images. From this perspective, these works about the figure ground segmentation and $3 \mathrm{D}$ reconstruction are also relevant [14][15].

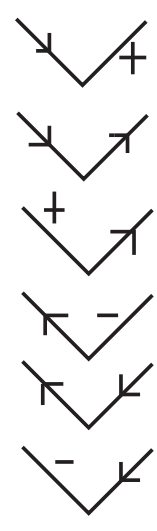

L
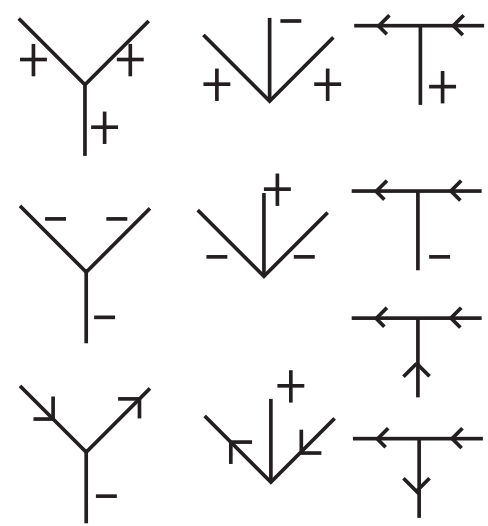

Y
W

W

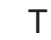

Fig. 1. Huffman-Clowes' catalogue for valid junctions. The plus sign stands for convex edges. The minus sign stands for the concave edges. The arrow indicates that an occluding surface is on the right side if we are moving towards the arrow's head.

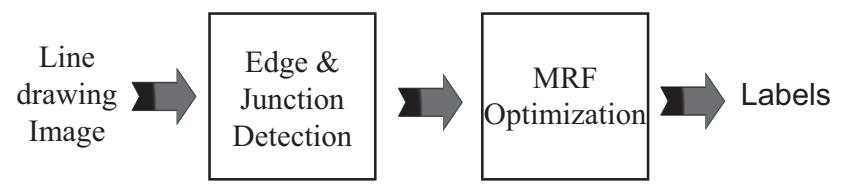

Fig. 2. The outline of our model.

\section{Description OF OUR MOdel}

The two modules of our model are shown in Figure 2. The first module extracts edges and junctions from the line drawing image, such as the cube in Figure 3. These images are typically clean. Therefore, the edge image can be obtained by thresholding the input image. Then, line segments are fitted using code from Kovesi's website [16]. After that, our model detects and classifies all the junctions in the image. Some simple rules are used to classify these junctions. E.g. if the junction only consists of two line segments, it has to be a $\mathrm{L}$ junction; if two of three line segments are almost collinear, it should be a $\mathrm{T}$ junction. Finally, this module determines the role of each edge for its connected junctions. E.g. we need to know which edge is the occluded stem of the $\mathrm{T}$ junction.

In the future we aim at extracting the contours of polyhedron in the natural images. This is more complicated, considering all kinds of noise in natural images. We also plan to enable this module to compute the likelihood of line labels based on local texture gradient, and to estimate the uncertainty related to each junction. In this paper, these two kinds of information are given by interaction.

The second module is responsible for choosing an optimal label for each edge. The MRF is constructed as a factor graph. Figure 4 is the MRF constructed from Figure 3. Accordingly, the BP algorithm for the factor graph is used as the inference engine. The solution may not be unique, especially when binary potentials are used. In other words, there is more than one valid labeling for the graph. In that case, BP algorithm will assign highest probability value to more than one label of some edges. If that happens, our model fixes an edge node to one of its feasible label and run BP again. If there are further ambiguities, this procedure is repeated. This is necessary to ensure the consistency of all the chosen labels. Note than, the model can keep track of all the choices that has been made to get an unique solution, therefore, our model is able to explore other choices and get other solutions.

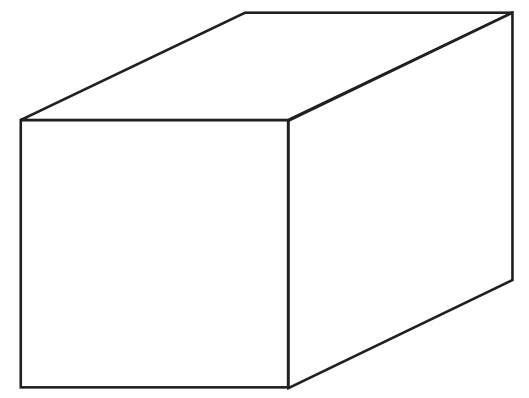

Fig. 3. An example of input line drawing image.

We assume that the probability of labeling $X$ is related to the energy of the MRF:

$$
\operatorname{Prob}(X) \propto \exp ^{-E(X, \sigma)}
$$

The energy function of the MRF is,

$$
E(X, \sigma)=-\prod_{i} \phi_{i}\left(x_{i}\right) \prod_{j} \psi_{j}\left(X_{j}, \sigma\right)
$$

where $X$ denotes the set containing all the edge labels $\left\{x_{i}\right\}$, $\phi_{i}\left(x_{i}\right)$ is the likelihood function for edge $i$, derived from 
image. $\psi_{j}\left(X_{j}, \sigma\right)$ is the potential for the junction $j, X_{j}$ denotes all the labels of the two or three edges connected to the junction $j . \sigma$ is a parameter between zero and one. It reflects the uncertainty and noise level of the detected junction.

Unlike many MRFs whose clique potentials are almost identical, these in our model need to be customized for every junction. First of all the type of the junction must be determined individually. Different junction type will surely give rise to different potential functions encoding the Huffman- Clowes catalogue shown in Figure 1. Second, different orders of edge nodes in $\psi$ will leads to different potential functions, because the potential functions are not symmetric. For example, in Figure 1, when the labels of two edges of an L junction are interchanged, the resulted labeling may not be valid. In our model, the edge variables always appear in ascending order of indices, therefore, the potential functions needs to be adjusted accordingly. Last, any arrow label can only point to one of the two junctions that edge connects. Therefore, the same label can mean either leaving or heading towards a junction. In our implementation, label 1 and 2 stands for convex and concave edges respectively. Label 3 stands for one of two directions along an edge; label 4 stands for the reverse direction of label 3. Take an L junction as example, we assume the label of the left edge is associated with rows of the matrix of potential function, and that of the right edge is associated with the columns of the matrix. If the labels 3 of both variables are towards the junction, the matrix of potential function should be as follows:

$$
\mathbf{M}=\left(\begin{array}{llll}
\sigma & \sigma & \sigma & 1 \\
\sigma & \sigma & 1 & \sigma \\
1 & \sigma & \sigma & 1 \\
\sigma & 1 & 1 & \sigma
\end{array}\right)
$$

Each valid labeling in Figure 1 give rise to the value ' 1 ' in the matrix, and the rest of configurations has a value of $\sigma$ which is less than one. We can see that when $\sigma=0$, the potential function is binary. In standard MRF formulation, the energy cannot be zero. However, it brings no problem to the BP. As discussed below, BP will simulate the edge consistency algorithm which is a classic solution to this problem. When $\sigma=1$, the potential function constantly equals one, which means all junction labeling are equally possible. This reflects the situation that the detected junction is extremely noisy.

The $\mathrm{Y}, \mathrm{W}$, and $\mathrm{T}$ junctions give rise to cubic matrix as potential functions, and they are constructed in a similar fashion as the L junction.

The update rule of belief propagation for a factored graph is as follows:

$$
\begin{gathered}
u_{\psi_{j} \rightarrow x_{i}}\left(x_{i}\right)=\max _{X_{j} \backslash x_{i}}\left(\psi_{j}\left(X_{j}, \sigma\right) \prod_{x_{k} \in X_{j} \backslash x_{i}} u_{x_{k} \rightarrow \psi_{j}}\left(x_{k}\right)\right) \\
u_{x_{i} \rightarrow \psi_{j}}\left(x_{i}\right)=\prod_{\psi_{k} \in N\left(x_{i}\right) \backslash \psi_{j}} u_{\psi_{k} \rightarrow x_{i}}\left(x_{i}\right) \cdot \phi_{i}\left(x_{i}\right)
\end{gathered}
$$

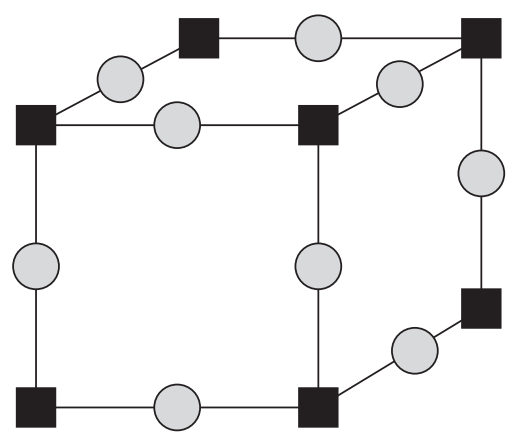

Fig. 4. The factor graph built on the line drawing of a cube. The black square denotes the junction potentials $\psi_{i}$ and the gray circle denotes the random variable which has four possible labels. The edge term $\phi_{i} \mathrm{~s}$ are not shown in this figure for clarity.

Where $u_{\psi_{j} \rightarrow x_{i}}\left(x_{i}\right)$ denotes the message from junction $j$ to the edge $i$, and $u_{x_{i} \rightarrow \psi_{j}}\left(x_{i}\right)$ denotes the message from the edge $i$ to the junction $j$. Initially, these messages are all set as constant, i.e. $u\left(x_{i}\right) \equiv 1$. In every iteration, the messages from the edge to the junction are updated using the messages from the junctions to the edges in the previous iteration, and vice visa. After the messages converge, the beliefs at each edge is computed as:

$$
b_{i}\left(x_{i}\right)=\prod_{\psi_{j} \in N\left(x_{i}\right)} u_{\psi_{j} \rightarrow x_{i}}\left(x_{i}\right) \cdot \phi_{i}\left(x_{i}\right)
$$

If the belief function of each edge has only one global maxima, the optimal solution $x_{i}^{*}$ is computed as:

$$
\forall i, x_{i}^{*}=\operatorname{argmax} b_{i}\left(x_{i}\right)
$$

However, if there are more than one global optimal solutions, the above equation can no longer lead to correct labeling. Our model solves this problem by fixing some of the edge label to one of the probable label until a unique solution is obtained.

BP algorithm is closely related to the edge consistency algorithm which is equivalent to the arc consistency algorithm [17]. This algorithm removes a label at one node if there are no compatible labels of adjacent nodes. Once the removal has been made, the change will be propagated to other nodes. This procedure is very similar to BPs message passing when $\sigma=0$ and $\forall i, \phi_{i}\left(x_{i}\right) \equiv 1$. From equation (4), we can see that $u_{\psi_{j} \rightarrow x_{i}}\left(x_{i}\right)$ equals one if and only if there are labels $x_{k},(k=$ $1 \ldots K)$ of adjacent nodes satisfying $\forall k, u_{x_{k} \rightarrow \psi_{j}}\left(x_{k}\right)=1$ and $\psi_{j}\left(x_{i}, x_{1} \ldots x_{K}\right)=1$. In other words, there must exist feasible labels $x_{k}$ of adjacent nodes which are compatible with $x_{i}$. If these compatible labels cannot be found, label will be considered as infeasible. Consequently, $u_{\psi_{j} \rightarrow x_{i}}\left(x_{i}\right)=0$, and equation (5) leads to $u_{x_{i} \rightarrow \psi_{j}}\left(x_{i}\right)=0$. Therefore the label cannot be used to support any label of adjacent node. For these reasons, the BP algorithm can be used to simulate the arc consistency algorithm. However, our model does not stop there. First, our model can pool in information from other sources via the edge term $\phi_{i}\left(x_{i}\right)$. Besides, the parameter $\sigma$ can reduce the negative impact of inaccurate junction detection, 
thus adds up to the robustness of our model. Therefore, our model can be considered as a soft representation of the Huffman-Clowess constraint.

\section{EXPERIMENTS}

The experiments consist of three parts. The first part validates out model with binary junction potentials and in absent of likelihood functions. It shows that our model could produce valid labeling to the line drawing images. In the second part, the likelihood functions are set to favor some valid labeling over others. This is to simulate the effect of likelihood function obtained from local image cues. In the third part, our model using the robust real-valued potential function can label the impossible line drawings as consistent as possible. There is no globally consistent labeling for these images. We assume that they are caused by erroneous junction detection. Therefore, we let these junction potentials have a positive $\sigma$. This parameter can encourage the model to assign inconsistent label to the junctions with maximal noise.

\section{A. Binary potentials without edge likelihood function}

The potential function is binary if $\sigma=0$. In this case, our model will simulate the arc consistency methods and produce valid labeling to each edge. We first demonstrate it on the cube shown in Figure 3. The result is shown in Figure 5. Note that

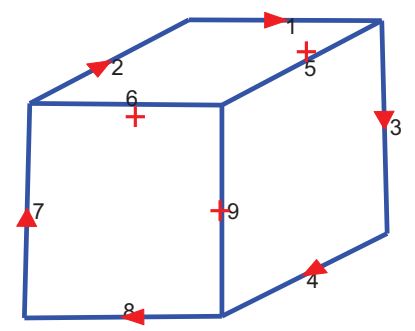

Fig. 5. Our models output for the image of cube in Figure 3. The blue lines are detected edges. The plus sign indicates a convex edge, the minus sign indicates a concave edge. The occluding surfaces are on the right of arrow.

this labeling is not the only one our algorithm can produce. After BP converged for the first time (after 3 iterations), the edge $1,2,3,4,7,8$ has two labels of with equal 'belief' (defined in equation 6). One of two labels is stands for the occluding edge in Figure 5. Another one stands for concavity '-'. The ambiguities arise because the context of the cube is unknown. For example, if the cube is placed on a desk, the edge 4 and edge 8 should have a label of concavity. In this experiment, our algorithm chooses the occluding edge over concave edge, whenever they have equal beliefs. The edges with two possible labels are disambiguated one by one. E.g. after fixing the label of edge 1, we run BP again. This time, BP solves the ambiguity of edge 3 . The rest of ambiguous labels are determined by repeating the above step. This procedure is necessary to ensure the consistency of labels. A more complex line drawing image is shown in Figure 6 and our models output is shown in Figure 7.

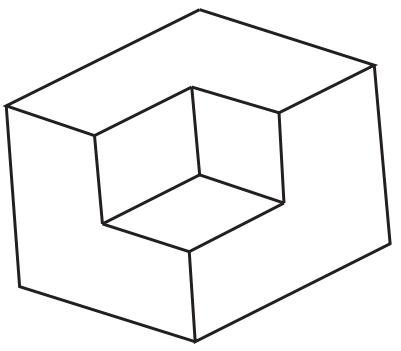

Fig. 6. An example of input line drawing image.

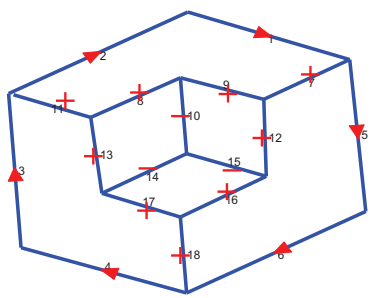

Fig. 7. Our models output for the line drawing in Figure 6.

\section{B. Binary potentials with edge likelihood}

In the last experiment, we assume that the object flows in the air, so that the occluding edge is preferred over concave or convex edge when they have the same belief value. However, in the natural images, there are many other cues that can help our model to disambiguate these labels. For example, the shadows in Figure 8 makes one believe that the cube is placed on a plane. In our model, this information can influence the labeling through the local edge likelihood function $\phi_{i}$ in equation 2 .

The cube in Figure 3 are again used in this experiment, the preference for the occluding edge is preserved. The difference is that this time we manually set the likelihood of convex and concave labels of edge 4 to 1 , and both occluding labels to 0.5 , i.e. $\phi_{4}(1)=\phi_{4}(2)=1, \phi_{4}(3)=\phi_{4}(4)=0.5$. The likelihood functions of other edges remain constant. We expect that our local detectors could produce similar values in the future. The output is shown in Figure 9. The edge 4 and edge 8 automatically obtain the label of concavity when the likelihood term of edge 4 slightly bias towards non-occluding labels. This demonstrates that our model can produce better labeling than the arc consistency methods with the help of likelihood function. 


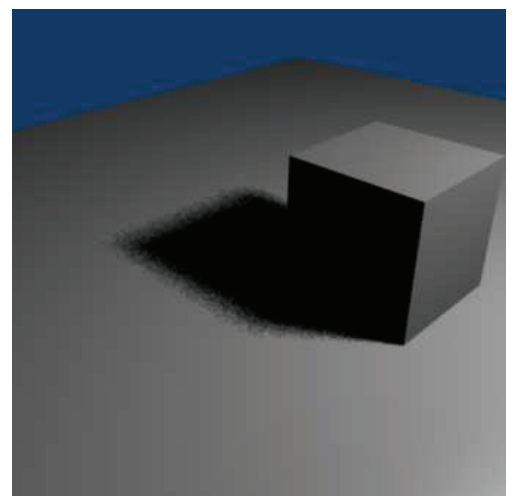

Fig. 8. The image of the cube may contain cues to disambiguate the labeling. For example, the shadow hints that the cube is placed on a plane.

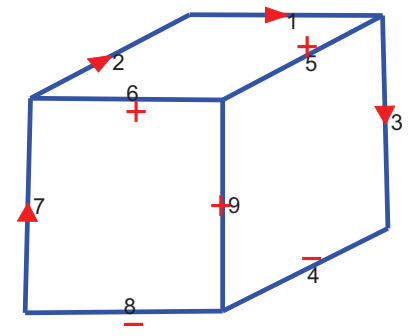

Fig. 9. Our models output under the influence of the likelihood function, which senses that the cube is placed on a plane.

\section{Real-valued potentials}

The constraints embedded in the Huffman-Clowes catalogue can be strictly enforced if there are no error in both edge and junction detection. In that case, our model shall use the binary potential functions which assign zero probability for invalid configuration. However, edge detection remains an open problem for natural images. Some edge may be missing; some spurious edge may emerge. Besides, the location and orientation of the edges may be inaccurate such that the junction types are misclassified. Sometimes a single incidence of local error could make the line drawings impossible to label. For example, the image in Figure 10 cannot be labeled consistently at every junction. When this happens, it is still desirable to label the edges as consistently as possible because the most of the junctions are correctly detected. Therefore, the labeling with minimal inconsistencies can still be meaningful. To achieve this goal, our model only needs to set $\sigma>0$ for all the junction potentials. The energy function (2) still favors model with minimal inconsistences, however, it has tolerance for imperfect labeling. Therefore, it can produce meaningful labeling for these impossible figure.

Our models output for the image in Figure 10 is shown in Figure 11. $\sigma$ of all potential functions are set to 0.1. All the junctions are consistently labeled except for one junction in the red circle. If $\sigma$ is set to 0 , it is impossible to get any solution since all the messages and beliefs equal to zero.

With equal $\sigma$ for every junction potential, the inconsistency of all junctions are treated equally. Our algorithm can do a better job if there are estimates about the noise level of each junction. It makes sense to encourage inconsistency to happen at the most noisy junctions. Accordingly, $\sigma$ of noisy junction should be larger than that of reliable junctions. Suppose we have the knowledge that the junction formed by edge $6,7,8$ in Figure 10 are less reliable than others. Then we let $\sigma=0.2$ for this junction and $\sigma=0.1$ for the rest of junctions. Our models output with these parameters is shown in Figure 12. The output is what we expected, the inconsistence only happens at the junction that is most likely to be erroneous (shown in red circle). Therefore, our model, with real-valued potential functions, is robust against erroneous junction detection.

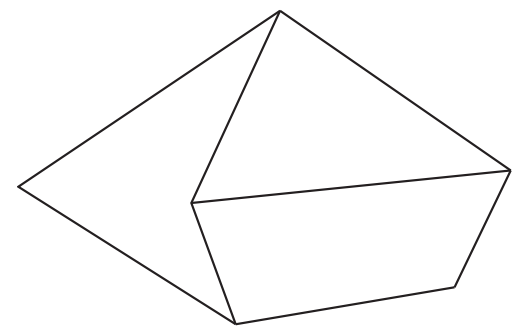

Fig. 10. A line drawing that is impossible to label correctly at every junction [2].

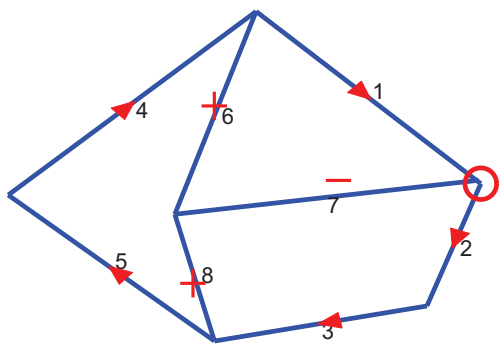

Fig. 11. Using the real-valued potential function, our algorithm is able to produce meaningful output with minimal local inconsistence. The invalid junction is in the red circle.

\section{CONCLusion And Future Work}

Interpretation of line drawings of trihedral objects is a classic topic in computer vision. In this paper, it is formulated as a Markov random field. Belief propagation algorithm is used for inference. With binary potential functions and constant likelihood functions, our model is very similar to the arc 


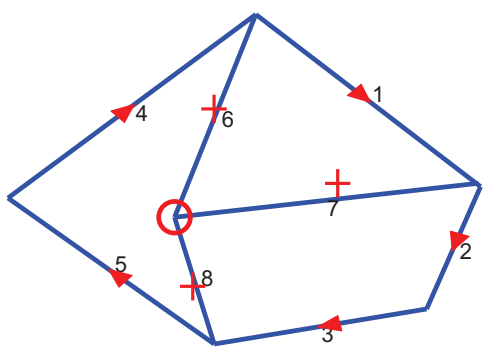

Fig. 12. By adjusting the of individual junctions, our model can encourage inconsistency to happen at the most noisy junction in the red circle.

consistency method. However, the experiments show that the likelihood function can integrate the information from other image cues; the real-valued potential function can make our model robust against errors in edge detection phase. These two advantages come from our probabilistic treatment of hard constraints.

In the experiment, we only tested our model on simple line drawing images because our algorithm, at current stage, cannot handle natural scene images with complex background and various illumination conditions. Correctly detection of edges and junctions in such images presents great difficulty and is beyond the reach of the simple edge and junction detection module which our algorithm is using. Nevertheless, we are working towards this end. Once the front-end detection has been improved, we plan to test our algorithm on natural scene images with polyhedral objects. For example, the algorithm could help a robot to understand some indoor environment. Besides, the edge and junction detectors should be able to estimate the noise level and uncertainty of each detected junction. We also plan to train classifiers based on local image cues to predict each edge label and transform their output into the likelihood function. Last, we will explore the possibility to extend our model to non-trihedral objects and scenes with shadow since they are commonplace in natural images [5].

\section{REFERENCES}

[1] B. Y. Liu, S. Gould, and D. Koller, "Single image depth estimation from predicted semantic labels," 2010 IEEE Conference on Computer Vision and Pattern Recognition (Cvpr), pp. 1253-1260 3632, 2010.

[2] D. A. Huffman, "Impossible objects as nonsense sentences," Machine Intelligence, vol. 6, pp. 295-323, 1971.

[3] M. B. Clowes, "Seeing things," Artificial Intelligence, vol. 2, no. 1, pp. 79-116, 1971.

[4] Y. Weiss and W. T. Freeman, "Correctness of belief propagation in gaussian graphical models of arbitrary topology," Neural Computation, vol. 13, no. 10, pp. 2173-2200, 2001.

[5] D. Waltz, Understanding line drawings of scenes with shadows. McGraw-Hill, 1975, pp. 19-91.

[6] A. K. Mackworth and E. C. Freuder, "The complexity of constraint satisfaction revisited," Artificial Intelligence, vol. 59, no. 1-2, pp. 5762, 1993.
[7] — " "The complexity of some polynomial network consistency algorithms for constraint satisfaction problems," Artificial Intelligence, vol. 25 , pp. $65-74,1985$.

[8] A. K. Mackworth, "Consistency in networks of relations," Artificial Intelligence, vol. 8, no. 1, pp. 99-118, 1977.

[9] T. Regier, "An approach to line labeling using markov random fields," in System Sciences, 1991. Proceedings of the Twenty-Fourth Annual Hawaii International Conference on, vol. i, pp. 514-522 vol. 1.

[10] J. Malik, "Interpreting line drawings of curved objects," International Journal of Computer Vision, vol. 1, no. 1, pp. 73-103, 1987.

[11] M. C. Cooper, "A rich discrete labeling scheme for line drawings of curved objects," Pattern Analysis and Machine Intelligence, IEEE Transactions on, vol. 30, no. 4, pp. 741-745, 2008.

[12] W. Whiteley, "The machine interpretation of line drawings - sugihara,k," Journal of Mathematical Psychology, vol. 31, no. 4, pp. 441-448, 1987.

[13] D. Marr, Vision: A Computational Investigation into the Human Representation and Processing of Visual Information. W. H. Freeman, 1983.

[14] D. Hoiem, A. A. Efros, and M. Hebert, "Recovering occlusion boundaries from an image," International Journal of Computer Vision, vol. 91 no. 3, pp. 328-346, 2011 .

[15] D. C. Lee, M. Hebert, and T. Kanade, "Geometric reasoning for single image structure recovery," in Computer Vision and Pattern Recognition, 2009. CVPR 2009. IEEE Conference on, pp. 2136-2143.

[16] P. Kovesi, "Matlab and octave functions for computer vision and image processing," http://www.csse.uwa.edu.au/ pk/research/matlabfns.

[17] A. K. Mackworth, "Interpreting pictures of polyhedral scenes," Artificial Intelligence, vol. 4, no. 2, pp. 121-137, 1973. 